\title{
A study of spectral curvature in the radio relic in Abell 4038 using the uGMRT
}

\author{
Ruta Kale, ${ }^{1 \star}$ Viral Parekh, ${ }^{2}$ and K. S. Dwarakanath ${ }^{2}$ \\ ${ }^{1}$ National Centre for Radio Astrophysics, Tata Institute of Fundamental Research, Post Bag 3, S. P. Pune University Campus, \\ Ganeshkhind, Pune 411007, Maharashtra, India \\ ${ }^{2}$ Raman Research Institute, C. V. Raman Avenue, Sadashivanagar, Bangalore 5600 80, Karnataka, India
}

Accepted XXX. Received YYY; in original form ZZZ

\begin{abstract}
The remnant radio galaxies in galaxy clusters are important sources of seed relativistic electron population in the intra-cluster medium (ICM). Their occurrence and spectral properties are poorly studied. In this work we present a broadband study of the radio relic in the galaxy cluster Abell 4038 using the Upgraded Giant Metrewave Radio Telescope (uGMRT). We present the uGMRT images in the bands $300-500 \mathrm{MHz}$ and $1050-1450 \mathrm{MHz}$ having rms noise $70 \mu \mathrm{Jy}_{\text {beam }}{ }^{-1}$ and $30 \mu \mathrm{Jy}_{\mathrm{beam}}{ }^{-1}$, respectively, that are the deepest images of this field so far. A spectral analysis of the relic over 300 - $1450 \mathrm{MHz}$ using images in sub-bands scaled to have constant fractional bandwidths to achieve a closely matched uv-coverage was carried out. The $100 \mathrm{kpc}$ extent of the relic is divided into Loop, Arc, Bridge and North-end. The Loop has a steep spectral index of $\alpha=2.3 \pm 0.2\left(S_{v} \propto v^{-\alpha}\right)$. The North-end has ultra-steep spectra in the range $2.4-3.7$. The Arc is found to skirt a curved region seen in the Chandra X-ray surface brightness image and the highest spectral curvature in it reaches $1.6 \pm 0.3$. We interpret the morphology and spectral properties of the relic in the scenario of an adiabatically compressed cocoon from the past activity of the Brightest Cluster Galaxy in the cluster. A comparison of the properties of the A4038 relic with a sample of 10 such relics is discussed.
\end{abstract}

Key words: acceleration of particles - magnetic fields - radiation mechanisms: non-thermal - galaxies:clusters:individual:Abell 4038 - galaxies: clusters: intra-cluster medium - radio continuum:general

\section{INTRODUCTION}

Clusters of galaxies contain among the largest pools of baryons in the Universe called the intra-cluster medium (ICM). The ICM contains thermal gas at temperatures $10^{7}-10^{8} \mathrm{~K}$ and is also known to be magnetized at $\mu \mathrm{G}$ levels. About a third of the most massive clusters, radio synchrotron sources of extents of Mpc, co-spatial with the $\mathrm{X}$-ray emission from the thermal gas or at the periphery are found (e. g. Brunetti \& Jones 2014, a review). These provide evidence for the presence of electrons with relativistic energies in the ICM. Since diffusion time of such electrons from a single source across the cluster is far longer than their radiative lifetimes, processes of re-acceleration are invoked (Jaffe 1977). The cluster-wide diffuse synchrotron sources called the radio halos are believed to originate from insitu re-acceleration of electrons via turbulence injected dur-

* E-mail: ruta@ncra.tifr.res.in ing cluster mergers (Schlickeiser et al. 1987; Petrosian 2001; Brunetti et al. 2004; Brunetti 2011; Brunetti \& Lazarian 2016). The arc-like radio sources at cluster peripheries are proposed to originate in the re-acceleration of mildly relativistic electrons at cluster merger shocks (e. g. Enßlin et al. 1998). The turbulent re-acceleration and diffusive shock acceleration at the weak shocks in the ICM are both inefficient processes requiring, a seed population of mildly relativistic electrons (e. g. Kang \& Jones 2007; Brüggen et al. 2012; Pinzke et al. 2013, 2017).

The sources of seed relativistic electrons could be the secondary electrons produced in the hadronic collisions (Brunetti 2011) or those injected by the cluster galaxies (Brunetti \& Jones 2014). The Active Galactic Nuclei (AGN) and radio galaxies inject relativistic plasma via jets during the active phase of the central supermassive blackhole. However the jets and lobes of radio galaxies will fade on the timescales of $10^{7}-10^{8}$ years unless there are mechanisms at work that re-energize them (e. g. Schlickeiser et al. 
1987). Adiabatic compression of remnant radio cocoons due to shocks and disturbances in the ICM has been proposed to revive the radio emission (Enßlin \& Gopal-Krishna 2001). Simulations of this process predict that the compressed cooon will be shred into filaments allowing dispersal of the plasma (Enßlin \& Brüggen 2002). A mechanism of gentle re-acceleration also has been proposed recently (van Weeren et al. 2017). Such remnants that show revival from their fading phase have also been referred to as radio "phoenixes" (Kempner et al. 2004) in the literature.

In this work we focus on remnant relics that could be important sources of seed relativistic electrons in the ICM. The detection of such remnants in clusters is challenging due to their low brightness and rare occurrence due to short radiative lifetimes. A sample of 13 such relics has been presented in Feretti et al. (2012) where these are classified as "roundish" relics to distinguish them from the arc-like and elongated relics associated with merger shocks. A further larger sample of "phoenix" candidates has been presented in Nuza et al. (2017) and recently a faint subsample of these relics has been presented by Wilber et al. (2018). Integrated spectra of such relics over broad ranges of frequencies have been measured for some of the relics (Slee et al. 2001; van Weeren et al. 2011b; Cohen \& Clarke 2011; Kale \& Dwarakanath 2012). Spectral index studies of a few relics with good resolution across the relic have also been carried out by combining multi-frequency radio data from one or more radio telescopes and have revealed the complex morphologies and spectra and allowed to estimate the life cycle of the relativistic plasma in them (e. g. Cohen \& Clarke 2011; Kale \& Dwarakanath 2012; Shulevski et al. 2015, 2017).

Here we present a broadband study of a remnant radio relic towards the galaxy cluster Abell 4038 (A4038, hereafter) using the Upgraded Giant Metrewave Radio Telescope (uGMRT). The uGMRT observations resulting in the deepest images towards this source are presented and used to measure spectral curvature across the extent of the relic. The radio morphology, spectra and the X-ray surface brightness map of the cluster together point to a scenario in which the relic originated from the compression of a radio cocoon left by the central galaxy. A sample of such relics is presented and the A4038 relic in comparison with others is discussed and the importance of spatially resolved wideband observations of these sources is emphasized. The paper is organized as follows: Sec. 2 describes the cluster Abell 4038 and the relic in it. The observations and data analysis are described in Sec. 3. The uGMRT images are presented in Sec. 4. The spectral curvature analysis is described in Sec. 5. The results are discussed in Sec. 6. The summary and conclusions are presented in Sec. 7 .

We have used $\Lambda$ CDM cosmology with $H_{0}=70 \mathrm{~km} \mathrm{~s}^{-1}$ $\mathrm{Mpc}^{-1}, \Omega_{\Lambda}=0.73$ and $\Omega_{m}=0.27$ in this work.

\section{ABELL 4038}

Abell 4038 (also known as Klemola 44) is a galaxy cluster of richness class 2 at a redshift of 0.03 having a bolometric Xray luminosity of $(1.900 \pm 0.025) \times 10^{44} \mathrm{erg} \mathrm{s}^{-1}$ (Mittal et al. 2011). The properties of the cluster are listed in Table 1.

A diffuse steep spectrum source towards this cluster was
Table 1. Properties of Abell 4038.

\begin{tabular}{ll}
\hline RA $_{\mathrm{J} 2000}$ & $23 \mathrm{~h} 47 \mathrm{~m} 43.2 \mathrm{~s}$ \\
DEC $_{\mathrm{J} 2000}$ & $-28^{\circ} 08^{\prime} 29^{\prime \prime}$ \\
Redshift $^{\dagger}$ & $0.02819 \pm 0.00055$ \\
$k T^{\dagger}$ & $2.69 \pm 0.43 \mathrm{keV}$ \\
$L_{[0.01-40] \mathrm{keV}}++$ & $(1.900 \pm 0.025) \times 10^{44} \mathrm{erg} \mathrm{s}^{-1}$ \\
$\mathrm{M}^{\ddagger}$ & $1.5 \pm 0.1 \times 10^{14} \mathrm{M}_{\odot}$ \\
\hline \multicolumn{2}{l}{ Sanders et al. (2011) ++ Mittal et al. (2011) } \\
\multicolumn{2}{r}{ Planck Collaboration et al. (2016) }
\end{tabular}

discovered by Slee \& Siegman (1983). In a further study at $1.4 \mathrm{GHz}$ they reported a radio relic of size $56 \mathrm{kpc}$, with polarization at $1.4 \mathrm{GHz}$ of $4.6 \pm 2.3 \%$ and a spectral index of $3.1^{1}$ (Slee et al. 2001). Further high resolution multi-frequency study of the relic was presented by Kale \& Dwarakanath (2012). The Giant Metrewave Radio Telescope (GMRT) observations at frequencies 150, 235 and $610 \mathrm{MHz}$ revealed new steep spectrum regions of the radio relic of extent $100 \mathrm{kpc}$ and possibly up to $200 \mathrm{kpc}$. The integrated spectrum of the relic was modeled in the framework of the theoretical model by Enßlin \& Gopal-Krishna (2001). The relic was found to be consistent with the model of a radio galaxy lobe that is energized by adiabatic compression due to a passing shock wave. It was clear from this study that the relic showed complex morphological and spectral features that were not resolved. With the new uGMRT observations we have performed a spatially resolved spectral study of the radio relic.

\section{OBSERVATIONS AND DATA REDUCTION}

An upgradation of the receivers of the GMRT is presently being carried out. The upgraded GMRT (uGMRT) will have new receivers providing a near-seamless frequency coverage between $50-1500 \mathrm{MHz}$ (Gupta et al. 2017). The observations towards A4038 were carried out in the shared risk category during the Cycle 31. The data were recorded in 2048 frequency channels and two polarizations (RR, LL) with a sampling time of 8 seconds and observing duration of 8 hours in the bands $300-500$ and $1050-1450 \mathrm{MHz}$ (Table 2). The flux calibrators, $3 \mathrm{C} 48$ and $3 \mathrm{C} 147$, were used to calibrate the bandpass and absolute flux density scale. The secondary calibrator, 0025-260, was used to calibrate the phases towards the target.

Data were analyzed using the AOFlagger (Offringa et al. 2012) and Common Astronomy Software Applications (CASA) packages (McMullin et al. 2007). After removing non-working antennas, we ran AOFlagger with default parameters on the whole data set. It removed channels and time periods affected by radio freqeuncy interference (RFI). In both the frequency bands, it flagged $\sim 25 \%$ data. For bandpass and complex gain calibration, we used the standard steps in CASA. The flux density of the flux calibrators was set according to

1 The spectral index $\alpha$ of the synchrotron spectrum is defined as, $S_{v} \propto v^{-\alpha}$, where $S_{v}$ is the flux density of the source at the frequency $v$. 
Table 2. Summary of the uGMRT observations.

\begin{tabular}{|c|c|c|c|c|c|c|c|}
\hline Project Code & Date & $\begin{array}{l}\text { Freq. Band } \\
\text { MHz }\end{array}$ & $\begin{array}{l}\mathrm{BW} \\
\mathrm{MHz}\end{array}$ & $\begin{array}{c}\text { Freq. res. } \\
\mathrm{kHz} \text { channel }^{-1}\end{array}$ & $\begin{array}{c}\text { Time } \\
\text { hr }\end{array}$ & $\begin{array}{l}\text { Beam } \\
" x^{\prime \prime}, \circ\end{array}$ & $\begin{array}{c}\sigma_{r m s} \\
\text { mJy beam }^{-1}\end{array}$ \\
\hline 31_067 & 06 Feb. 2017 & $300-500$ & 200 & 97.7 & 8 & $10.0 \times 5.0,14.1$ & 0.07 \\
\hline 31_067 & 12 Mar. 2017 & $1050-1450$ & 400 & 195.3 & 8 & $3.6 \times 1.74,37.6$ & 0.03 \\
\hline
\end{tabular}

Perley \& Butler (2013). The AOFlagger was run on the calibrated visibilities. The target source (A4038) visibilities were then split after averaging of 10 channels. AOFlagger was used on these data to remove any remaining bad data before proceeding for imaging. The images were made using the final visibilities and standard steps of self-calibration. The multi-term multi-frequency synthesis mode (MT-MFS) in the CASA task clean was used to produce the final images. For the spectral index analysis we used a new method that ensured closely matched uv-coverage across the $0.402-1.4 \mathrm{GHz}$ (Sec. 5).

\section{UGMRT IMAGES OF A4038}

The uGMRT images at the effective frequencies of $402 \mathrm{MHz}$ and at $1.26 \mathrm{GHz}$ are shown in Fig. 1. The radio relic in A4038 is at the centre of the field. The detection of diffuse steep spectrum lobes of a radio galaxy in the $402 \mathrm{MHz}$ image is also marked (Appendix A).

The $402 \mathrm{MHz}$ image of A4038, overlaid on the Chandra X-ray image (OBSID 04992, $0.5-7 \mathrm{keV}$ ) is shown in Fig. 2. The different parts of the extended radio relic are labelled as the Loop, Arc, North-end and Bridge. The Loop and the Arc are well resolved at $1.26 \mathrm{GHz}$ and fall to the west of the BCG and do not show any obvious connection to the galaxies in that region (Fig. 3). There are two discrete sources in the cluster region labeled A4038_11 and A4038_10 (Fig. 2). The source A4038_11 is associated with the brightest cluster galaxy (BCG) in the cluster as seen in the optical band (Fig. 3). The second discrete source A4038_10 is likely a distant source as no optical counterpart can be seen.

The overall morphology of the relic is filamentary. The Arc and the Loop are the brightest regions of the relic that are detected across all the wavelengths. The Loop is resolved at $1.26 \mathrm{GHz}$ (Fig. 3, right) and reveals the knot-like structure that makes the southern brightest region of the relic. At lower frequencies a region labeled as the "Bridge" between A4038_11 and the Arc is detected (Fig. 2). The Bridge is co-spatial with the brightest region in X-rays that is also elongated in the same direction as the Bridge. In addition, an extended part of the Arc towards the north turns west and terminates at a brighter but, diffuse, region labeled the "North-end". An extension, further towards the north-west, that was speculated based on $240 \mathrm{MHz}$ image by Kale \& Dwarakanath (2012), is not detected, and was an artifact. At $402 \mathrm{MHz}$ the radio relic has an angular extent of $3^{\prime}(102 \mathrm{kpc})$ in the north-south direction and a largest width of $1.7^{\prime}(58 \mathrm{kpc})$ in the east-west.

The flux densities of the discrete sources A4038_11 and A4038_10 were measured from the images made with the highest resolution in order to minimize the contamination due to the radio relic. A4038_11 has a flux density of $51 \pm 2$ mJy at $402 \mathrm{MHz}$ and $31 \pm 3 \mathrm{mJy}$ at $1.26 \mathrm{GHz}$ and A4038_10 has a flux density of $10 \pm 1 \mathrm{mJy}$ and $3.3 \pm 0.3 \mathrm{mJy}$ at the respective frequencies. The flux densities for these discrete sources are reported based on the Gaussian fit obtained to the detected source assuming a single component.

\section{SPECTRAL CURVATURE}

\subsection{Matched uv-data}

The wideband observations were used to analyze the continuous variation of the spectral index across the frequencies and across the spatial extent of the relic. The RFI typically severely affects the short baselines which are crucial for imaging extended sources. Since our focus of study is the extended radio relic, we chose the regions of bandwidth that had suffered minimal flagging at short baselines. This resulted in the choice of frequency ranges from $380-460 \mathrm{MHz}$ in Band 3 and $1300-1440 \mathrm{MHz}$ in Band 5 for this analysis. Furthermore, to study the continuous spectral variation, it is important that our sampling in the uv-plane be similar in the frequency ranges that we consider. We fixed a bandwidth of $11 \mathrm{MHz}$ at $380 \mathrm{MHz}$ for a secure detection of the relic, and split the data into measurement sets with narrow bandwidths keeping the $\Delta v / v=0.028$ constant over the range of frequencies between $380-1440 \mathrm{MHz}$. This ensures that the width of the uv-track is the same across the bands to match the uv-coverage. This results in sub-band measurement sets with increasing bandwidth as a function of frequency. In our case the bandwidths range from $11 \mathrm{MHz}$ at the lowest to $40 \mathrm{MHz}$ at the highest frequency end. These bandwidths thus represent the limits below which the spectrum is assumed to be straight. The images were separately made from each of the measurement sets using "uniform" weights (robust $=-2.0$ ) for the visibilities. The uv-range while imaging is also restricted to the range, $200-31000 \lambda$, which is the overlapping uv-range across the selected frequency bands. These images produced with closely matched uv-coverage were inspected and those with a poor rms noise were excluded from the analysis. The final images were convolved to a common resolution of $10.4^{\prime \prime} \times 10.4^{\prime \prime}$ and used for the spectral analysis. The images from individual sub-bands and those from the legacy GMRT are shown in Fig. 4.

\subsection{Spectra}

The relic was divided into regions of size $15^{\prime \prime} \times 15^{\prime \prime}$ for spectral curvature analysis (Fig. 5). This size was chosen to be larger than the beam so that independent regions are used for spectral analysis. The selected regions cover the North 

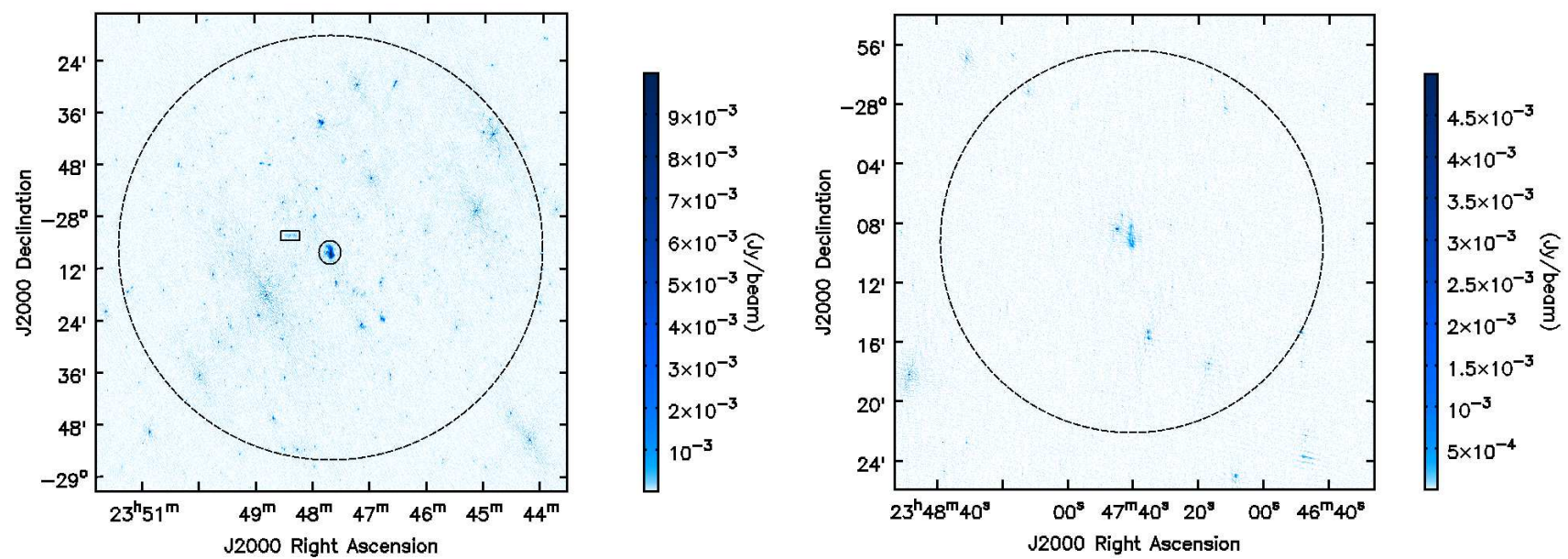

Figure 1. Left:- The uGMRT $402 \mathrm{MHz}$ image using data between $300-500 \mathrm{MHz}$. The rms is $0.07 \mathrm{mJy}$ beam ${ }^{-1}$ near the centre of the field. The central ellipse shows the relic in A4038 and the rectangle marks the detection of diffuse lobes of a radio galaxy. Right:- The uGMRT $1.26 \mathrm{GHz}$ image using data between $1050-1450 \mathrm{MHz}$. The rms is $0.03 \mathrm{mJy}$ beam ${ }^{-1}$ near the centre. The synthesized beams are reported in Table 2. In both the panels, circles with diameters equal to the respective primary beam half power widths are shown.
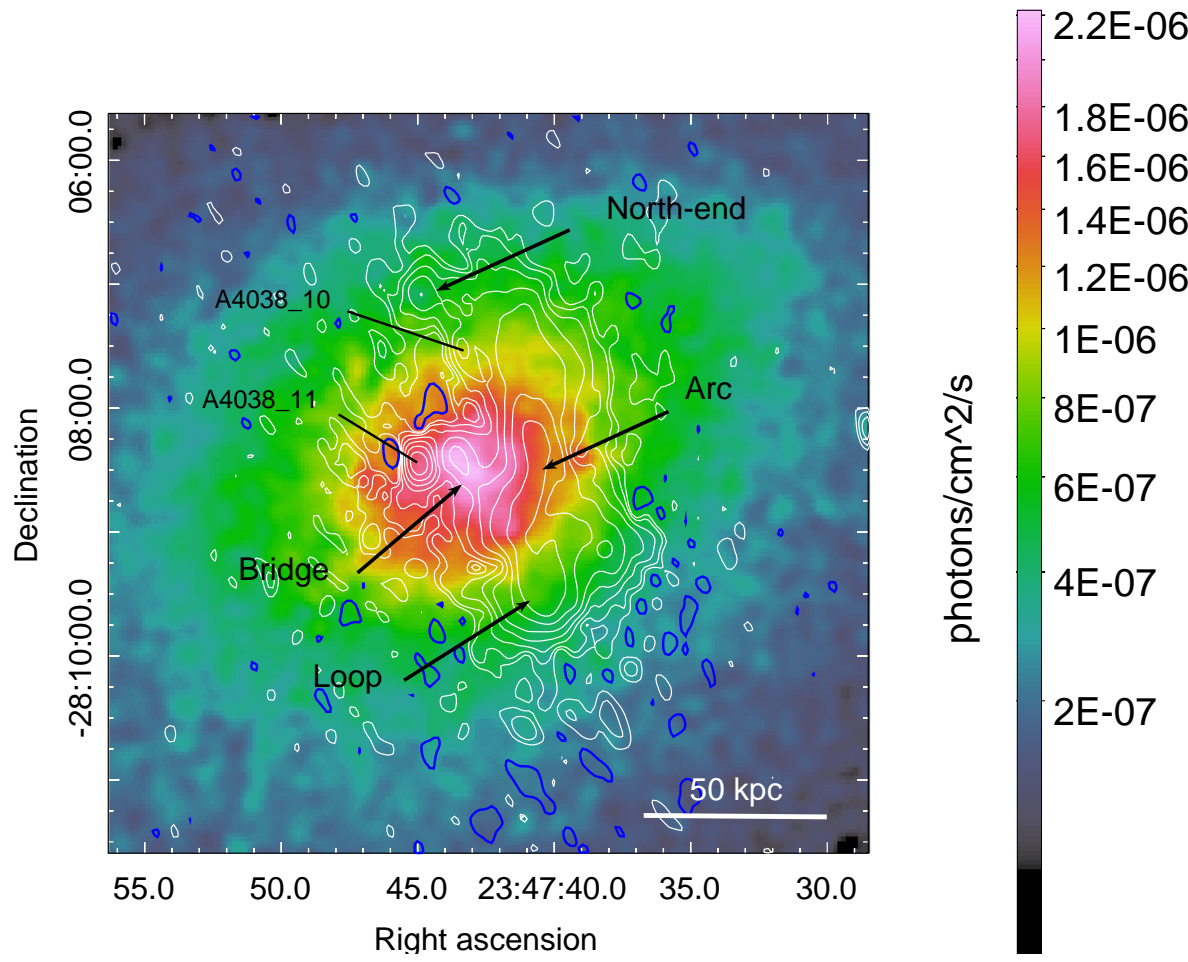

Figure 2. The uGMRT $402 \mathrm{MHz}$ image is shown in contours overlaid on the Chandra $0.5-7 \mathrm{keV}$ X-ray image (OBSID 04992) in colour. The contour levels start at $\sigma \times[-3,3,6,12, \ldots]$, where $\sigma=0.07 \mathrm{mJy}$ beam $^{-1}$ is the rms noise. Positive contours are white and the negative are blue. The discrete sources are A4038_11 and A4038_10. The parts of the radio relic are labelled "Arc", "Loop" and "Bridge".

end (0), the northern part of the relic $(1-5)$, the Arc $(6$ $9)$ and the Loop $(10-13)$. The region number 14 covers the Bridge. The flux densities in these regions were extracted from the images presented in Fig. 4. For the regions $0-3$ a single power-law was fit to the measurements at low frequencies. In the sub-band images at high frequencies, these regions were not detected due to their steep spectra. From region 4 to 14 the a separate power-law fits were made in the frequency ranges $0.32-0.45 \mathrm{GHz}$ and $0.45-1.43 \mathrm{GHz}$.
The linear fits were of the form,

$\log \mathrm{S}=-\alpha_{\operatorname{low}} \log v+\mathrm{B}$.

For the high frequency range the derived parameters were $\alpha_{\text {high }}$ and $C$. These are listed in Table 3. The spectra for the regions are plotted in Fig. 6 (left).

The difference, $\Delta \alpha=\alpha_{\text {high }}-\alpha_{\text {low }}$ provides a measure of the spectral curvature and is plotted for each of the regions in Fig. 6. The curvature is positive in all the regions with variation between 0.0 - 1.6. Along the Arc from north to 

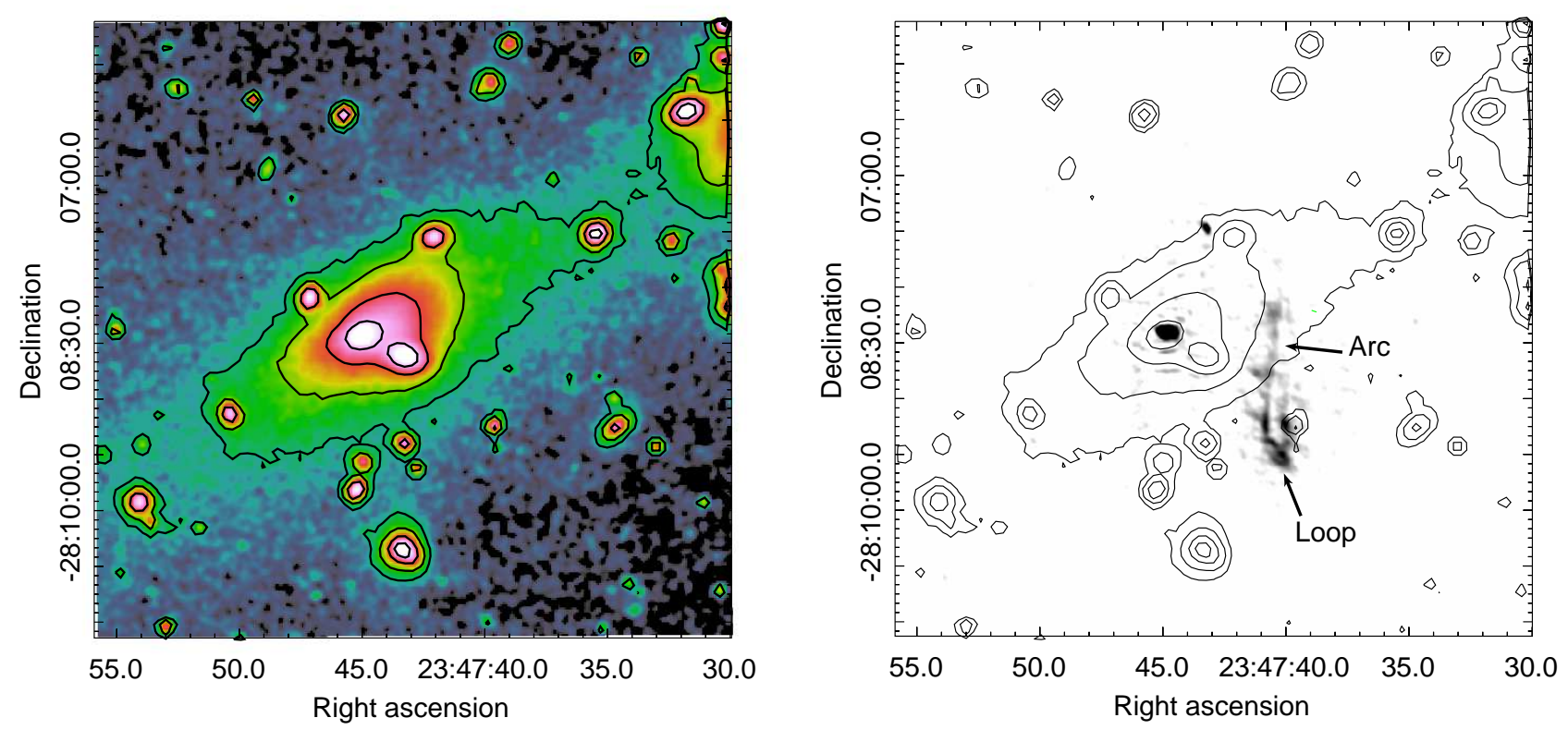

Figure 3. Left:- Digitized Sky Survey POSS II R-band optical image of A4038 is shown in colours and contours. Right:- The 1.26 GHz image with resolution $3.6^{\prime \prime} \times 1.47^{\prime \prime}$, p. a. $37.6^{\circ}$ is shown in grey scale and the optical contours same as those in the left panel are overlaid. The "Loop" and the "Arc" are labelled.

south, the curvature increases from 1 to 1.6 and then decreases in the Loop. The regions 0 - 3 need high sensitivity measurements above $0.6 \mathrm{GHz}$ to quantify their spectral shapes.

\section{DISCUSSION}

We have presented first observations of the cluster A4038 with the uGMRT in bands $3(300-500 \mathrm{MHz})$ and $4(1050$ - $1450 \mathrm{MHz})$. The cluster has a central radio source associated with the BCG and a peculiar, extended ultra- steep spectrum radio relic located adjacent to the core. The rich spatial and spectral structure in the radio relic is analyzed using the uGMRT observations.

\subsection{Equipartition magnetic field}

Direct estimate of magnetic fields towards such relics is difficult. Using the measurements in radio bands, the magnetic field assuming equipartition can be calculated. Following, Govoni \& Feretti (2004), the minimum energy density $u_{\min }$ is given by,

$$
\begin{array}{r}
u_{\min }\left[\frac{\mathrm{erg}}{\mathrm{cm}^{3}}\right]=\xi\left(\alpha, v_{1}, v_{2}\right)(1+k)^{4 / 7}\left(v_{0[\mathrm{MHz}]}\right)^{4 \alpha / 7} \times \\
(1+z)^{(12+4 \alpha) / 7}\left(I_{0\left[\frac{\mathrm{mJy}}{\operatorname{arcsec}^{2}}\right]}\right)^{4 / 7}\left(d_{[\mathrm{kpc}]}\right)^{-4 / 7}
\end{array}
$$

where $k$ is the ratio of energy in relativistic protons to that in electrons, $\alpha$ is the synchrotron spectral index, $v_{0}$ is the frequency at which the surface brightness, $I_{0}$ is measured, $d$ is the depth of the source and $\xi\left(\alpha, v_{1}, v_{2}\right)$ is a parameter that is a function of the spectral index and the lower and higher limits in frequency, $v_{1}$ and $v_{2}$ (Govoni \& Feretti 2004). In this formulation, the $K$-correction is included and a filling factor of 1 is assumed. The magnetic field is then given by,

$B_{\text {eq }[\mathrm{G}]}=\left(\frac{24 \pi}{7} u_{\min }\right)^{1 / 2}$.

For A4038 relic we have $d=80 \mathrm{kpc}$ which is the average of the projected extents 102 and $56 \mathrm{kpc}$. At $v_{0}=402.0 \mathrm{MHz}$ we measure $I_{0}=0.0829 \mathrm{mJy} \operatorname{arcsec}^{-2}$ for A4038 relic. The modified equipartition magnetic field $\left(B_{\text {eq }}^{\prime}\right)$ based on a limit on the minimum Lorentz factor, $\gamma=100$ rather than on the frequency (e.g. Brunetti et al. 1997; Beck \& Krause 2005) is given by,

$B_{\mathrm{eq}[\mathrm{G}]}^{\prime} \sim 1.1 \gamma_{\mathrm{min}}^{\frac{1-2 \alpha}{3+\alpha}} B_{\mathrm{eq}}^{\frac{7}{2(3+\alpha)}}$,

where $B_{e q[\mathrm{G}]}$ is from Eq. 3 .

The minimum energy and the magnetic fields determined for A4038 parameters as a function of the spectral index are shown in Fig. 7. For A4038 the integrated spectral index in the range $0.074-0.327 \mathrm{GHz}$ is 1.5 and steepens at higher frequencies (Kale \& Dwarakanath 2012). At $\alpha=1.5$ we find the $\mathrm{B}_{\text {eq }}$ and $\mathrm{B}_{\text {eq }}^{\prime}$ to be 3.3 and $7.8 \mu \mathrm{G}$, respectively.

In the above formalism it has been assumed that the spectrum is a single power-law across the range of frequencies. However the integrated spectrum of A4038 relic is not a single power-law. Moreover the spectra are curved also within the relic. Therefore the standard formalism is insufficient to calculate the magnetic field in such systems. The occurrence of curvature in the spectrum may be due to superposition of distinct power-law spectra within the smallest region considered here to measure the spectrum. This can be checked with more sensitive and higher resolution observations. A radically different possibility is of an intrinsically curved electron energy distribution that has been pro- 


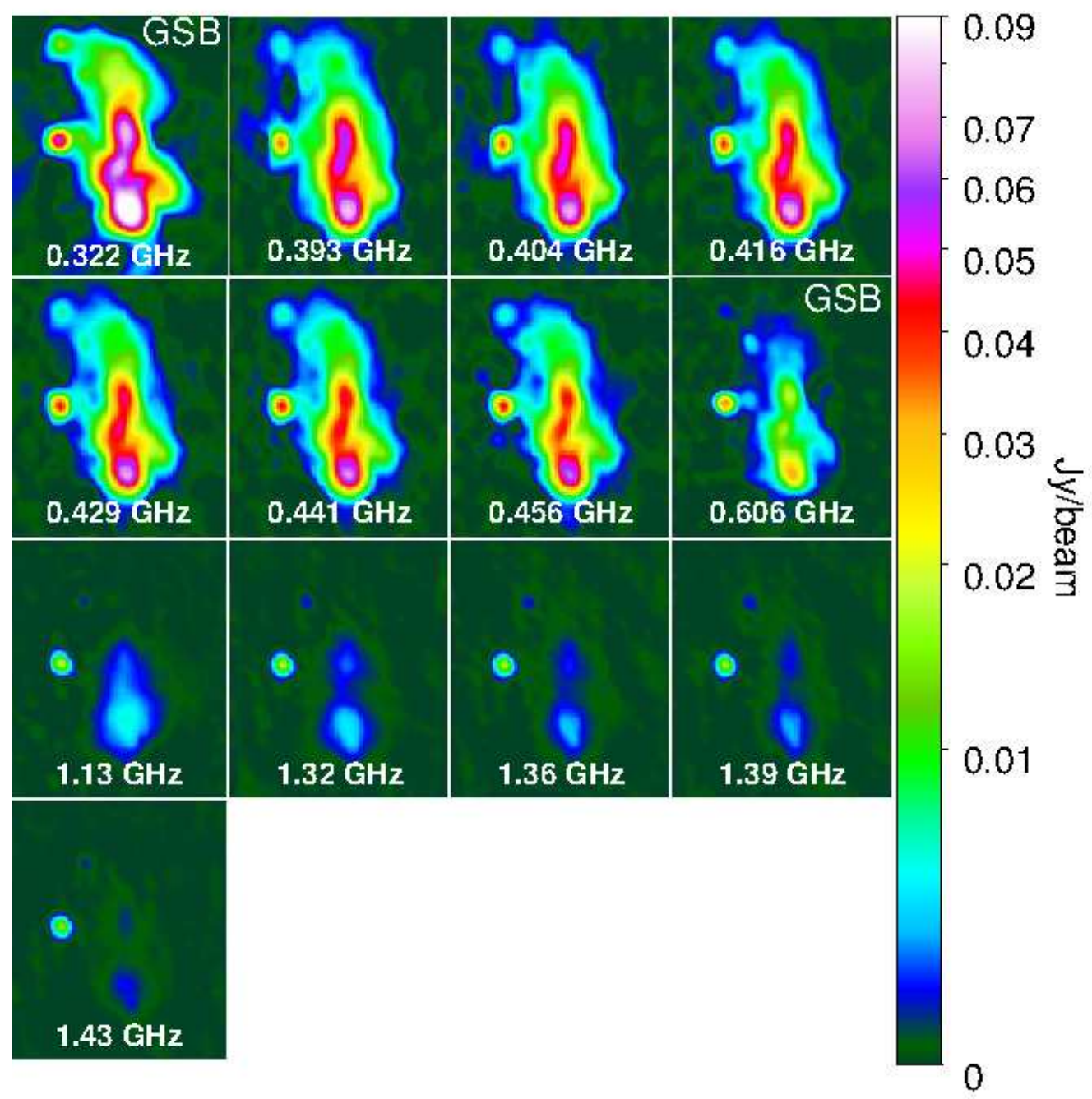

Figure 4. The images of A4038 relic at each of the narrow bands used for the spectral analysis. The two with the legacy GMRT system are marked as "GSB". The remaining are from the data from the uGMRT. The colour scale in the range $0.00-0.09 \mathrm{Jy}^{\text {beam }}{ }^{-1}$ is common for all the panels. All the images have been convolved to the common resolution of $10.4^{\prime \prime} \times 10.4^{\prime \prime}$.

posed to explain the curved spectra of radio galaxy lobes (Duffy \& Blundell 2012).

\subsection{Relic and X-ray connection}

In the case of diffuse radio sources it is non-trivial to make the association with either the X-ray emission or any optical counterpart. For A4038 relic we examined the evidence for connection with the ICM of the cluster. The BCG in the cluster (IC5358) is a radio galaxy located at an offset of 10 $\mathrm{kpc}$ from the peak in X-rays. The brightest region of X-rays is elongated in the northeast - southwest direction for 20 $\mathrm{kpc}$, and the Bridge is co-spatial with it. The Arc region of the relic skirts a curved region in X-rays that extends to the north as shown in Fig. 8. The steeper northern parts of the relic extend beyond the X-ray arc. From the morphology, we infer that the Arc of the relic is near the cluster core and the rest of the relic extends beyond it. As noted by Slee et al. (2001), there appears to be no connection between the optically detected galaxies and the relic.

A spectral index map between 606 and $240 \mathrm{MHz}$ with a resolution of $25^{\prime \prime}$ was presented in (Kale \& Dwarakanath 2012). However that did not resolve the Arc and the Loop. Here the spectral curvature across the relic is resolved using the uGMRT observations. We find that the integrated spectrum of the relic is not made up of self-similar spectral regions across the relic but shows significant variation in curvature (Fig. 6, right). The high frequency spectral index shows low variation though overall it is steep; it is the low frequency spectral index that shows flattening in the Arc region. Curved spectra and filamentary morphology are strong signatures for an origin in compression (Enßlin \& Brüggen 2002). The high curvature at the Arc region implies proximity of it to the disturbance that caused the compression. The presence of the Bridge (region 14) region supports a 


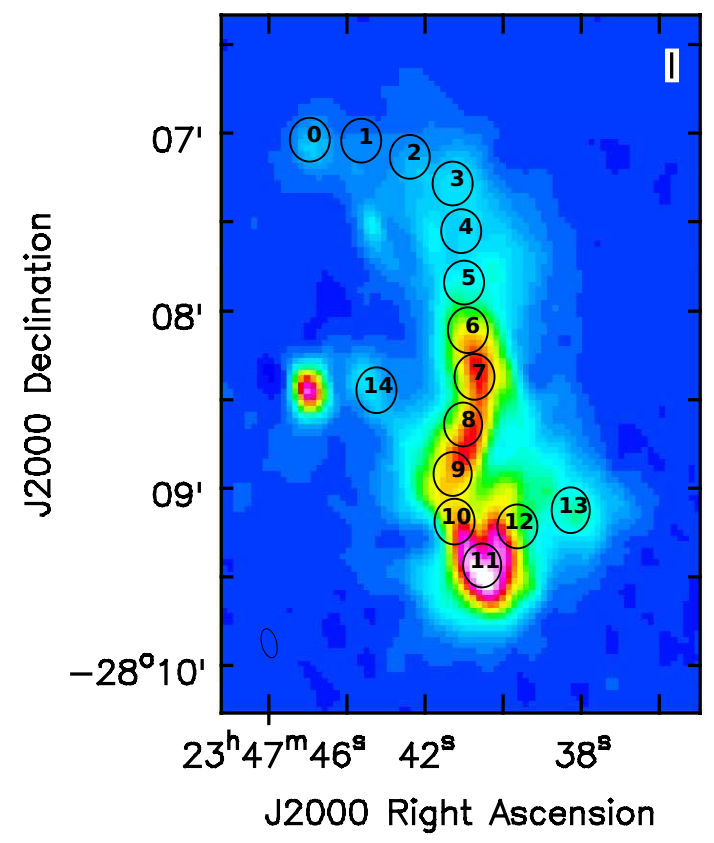

Figure 5. The regions in which the spectral curvature analysis of the relic is carried out are marked on the $402 \mathrm{MHz}$ image.

scenario where there is a connection between the relic and the BCG. The relic may have originated from the cocoon of the past activity of the BCG.

There is evidence for the disturbance at the cluster centre from the detection of turbulent broadening of spectral lines in X-rays. Turbulent broadening in line widths of 1300 $\mathrm{km} \mathrm{s}^{-1}$ at the cluster core has been reported (Sanders et al. 2011), which implies that it does not have a cool core. This supports the possibility of the disturbance at the core that displaced the BCG away from the X-ray peak and led to the compression of the radio plasma. A deeper investigation of the $\mathrm{X}$-ray emission is required to find morphological features that correlate with the radio relic.

\subsection{Comparison with other relics}

Remnants of radio galaxies in general are not limited to be in galaxy clusters. From the samples of radio sources with steep spectra, possible remnants of double radio sources have been discussed (Dwarakanath \& Kale 2009; van Weeren et al. 2011b; Shulevski et al. 2015). Such remnants need not necessarily be steep spectrum sources (Brienza et al. 2017). Several clusters with shock-related radio relics and central radio halos show presence of other smaller scale diffuse emission (e. g. van Weeren et al. 2016). In order to carry out a fair comparison between relics, a sample of objects with possible common origin are needed. Here we specifically discuss remnant sources that have been found in confirmed clusters of galaxies that contain ICM detected in X-rays and do not have other large-scale complex diffuse radio sources. We selected our sample of cluster remnant relics starting from the samples of relics presented in Feretti et al. (2012), van Weeren et al. (2011a,b) and Nuza et al. (2017). Our sample is presented in Table 4. The integrated spectral indices in low and high frequency bands are compiled from the literature. The relics in A1367 and A610 are not considered due to lack of spectral and morphological information. A2256 relic may have contributions due to direct shock acceleration at the relic and is thus excluded (Trasatti et al. 2015). According to the classification by Nuza et al. (2017), there are five confirmed phoenixes, namely, A85, A2048, A2443, A4038 and 24P73. We excluded the relic $24 \mathrm{P} 73$ due to the absence of confirmed diffuse X-ray emission from the associated galaxy cluster.

The remnant relics are typically irregular in appearance. The mean distance of the sample remnant relics from their host cluster centres is $0.36 \mathrm{Mpc}$, with A4038 being the one closest to the cluster centre. The morphology and spectra of these relics indicate a high chance that these are revived remnants or phoenixes. The host clusters are all nearby, ranging in redshifts from $0.01-0.13$ and have a mean redshift of 0.07 . This is not surprising as the lifetimes of the remnants would reduce with increasing redshift due to the inverse Compton losses to Cosmic Microwave Background photons $\left(t_{\text {loss }} \propto(1+z)^{-4}\right)$. Since the sample is not from a complete sample of clusters across redshifts, the occurrence of such relics cannot be judged yet. The location of the relics relative to the cluster centers is in the range $0.02-0.5 \mathrm{Mpc}$ and shows no trend with redshift; A1664 is the only outlier at a distance of $1 \mathrm{Mpc}$ from the cluster centre.

From the integrated spectra reported in the literature, the low and high frequency spectral indices were determined and are reported in Table 4 . We do not find any trend between the distance of the relic from the cluster centre and the spectral indices or the curvatures. The curvatures are all greater than 0.5 with A85 relic showing the highest curvature of 1.3. In Fig. 9, the low and high frequency spectral indices for the sample relics derived from the integrated spectra are shown. The deviation above the dashed line $\left(\alpha_{\text {low }}=\right.$ $\left.\alpha_{\text {high }}\right)$ indicates a positive curvature in the spectrum. The relic A1664 is an outlier among the considered relics with the flattest spectra. The rest of the sample has spectra steeper than 1.5 and are thus either in the passively evolving remnant phase or have been revived by mechanisms other than direct injection by an AGN. These mechanisms include adiabatic compression by ICM shocks and gentle re-acceleration processes (Enßlin \& Gopal-Krishna 2001; van Weeren et al. 2017). The spectral indices across the A4038 relic presented with wideband observations show values steeper than 1.5 and a variation in the range $1.2-3.7$. The integrated spectrum is thus composed of low and high brightness regions with a wide variety of spectral curvatures that are not well represented by the curvature of the integrated spectrum. It is essential to carry out resolved studies such as the one presented for A4038 relic here. The uGMRT is an ideal instrument to carry out these studies for samples of relics. Studies with closely matched observations between X-rays and radio are needed in order to find the phenomena behind the formation of the remnant relics in galaxy clusters.

\section{SUMMARY AND CONCLUSIONS}

We have presented a spectral curvature study of the relic in Abell 4038 using the uGMRT in the $300-500 \mathrm{MHz}$ and 1050 - $1450 \mathrm{MHz}$ bands. The connection between the relic properties and the X-ray emission from the cluster was discussed. 

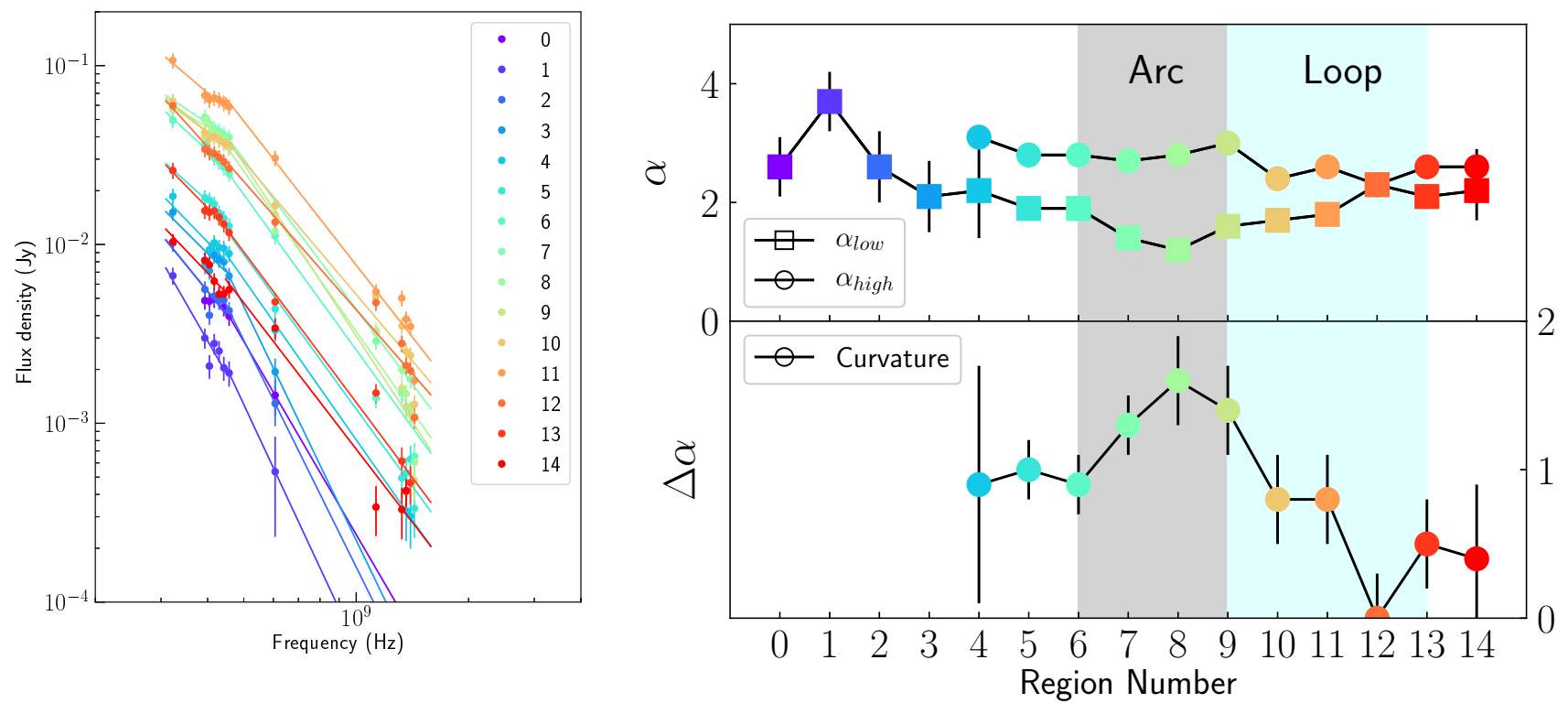

Figure 6. Left:- The spectra of the regions marked in Fig. 5 are plotted with a range of colors as shown in the legend. The best fit power-law spectra in the low and high frequency range are shown with solid lines. Right:- In the top panel, spectral indices $\alpha_{0.32}^{0.45}$ and $\alpha_{0.45}^{1.43}$ are plotted as a function of the region number shown on the x-axis. The difference between the two spectral indices (curvature) as a function of the region number is shown in the bottom panel. The grey shaded region marks the region numbers corresponding to the Arc and the cyan shaded region marks the Loop. To the right of the Loop is the region 14 corresponding to the Bridge. To the left of the Arc are regions in the northern part that are detected only at low frequencies and thus have no higher frequency spectral index and curvature measurements. The color scheme for the region numbers is the same as in the left panel.

A comparison between the A4038 relic and other small scale cluster relics was also carried out. The main findings are summarized below:

(i) We have produced deep images of the radio relic in A4038 at 0.402 and $1.26 \mathrm{GHz}$ using the uGMRT with bandwidths of 200 and $400 \mathrm{MHz}$ in the two bands, respectively. The rms noise at the centre of the field at $402 \mathrm{MHz}$ was 0.07 mJy beam ${ }^{-1}$ and at $1.26 \mathrm{GHz}$ was $0.03 \mathrm{mJy}_{\text {beam }}^{-1}$. The largest extent of the relic in the north-south direction is 102 $\mathrm{kpc}$ and in the east-west direction is $58 \mathrm{kpc}$.

(ii) The spectra across the relic were estimated in 15 regions, each of size $15^{\prime \prime} \times 15^{\prime \prime}$. The spectrum of each region was fit with separate power-laws in the ranges $0.32-0.45$ and $0.45-1.43 \mathrm{GHz}$. The difference in the spectral indices in the low $\left(\alpha_{\text {low }}\right)$ and high frequency $\left(\alpha_{\text {high }}\right)$ ranges was used as a measure of the spectral curvature $(\Delta \alpha)$.

(iii) The highest curvature, $\Delta \alpha$, of $1.6 \pm 0.3$ was found in the region corresponding to the Arc. The curvature is 0.8 in the regions 10 and 11 that make the eastern and southern parts of the Loop, respectively. To the west of the Loop it becomes consistent with zero in region 12 and then rises to 0.5 further west.

(iv) A curved arc-like region in the X-rays is skirted by the Arc in the relic. We propose that the highly curved spectra in the Arc result from compression-revived radio emission. This is consistent with our earlier work presented in Kale \& Dwarakanath (2012), where the integrated spectrum was best fit in the adiabatically compressed phase in the model by Enßlin \& Gopal-Krishna (2001).

(v) The calculation of magnetic field under the minimum energy condition is dependent on the spectral index and the current formalisms assume a single power-law. For A4038 relic if we assume a spectral index of 1.5, the magnetic field is $7.8 \mu \mathrm{G}$ if the minimum Lorentz factor, $\gamma_{\min }=100$.

(vi) We also present a sample of 10 remnant relics in galaxy clusters that have detectable X-ray emission from their ICM. These relics are typically found within the 0.5 Mpc from the cluster centre-the only exception being A1664.

(vii) We have plotted low versus high frequency spectral indices for the remnant relic sample, together with the values for the A4038 relic. Except the A1664 relic which has a flat and straight spectrum, the sample remnant relics have curvatures in the range $0.5-1.6$. These are consistent either with an old synchrotron plasma or a plasma re-energised by a mechanism such as adiabatic compression by shock or gentle re-acceleration.

(viii) We conclude that for A4038 the spectral shape changes across the regions within the relic and thus spatially resolved spectral studies as presented for the case of A4038 relic are essential to study the origins of such relics.

(ix) The deep radio images from the uGMRT presented here also resulted in the detection of faint lobes around a radio core identified with the galaxy PGC072471.

\section{ACKNOWLEDGEMENTS}

We thank the anonymous referee for critical comments that have improved this paper. RK acknowledges support through the DST-INSPIRE Faculty Award by the Government of India. We thank the staff of the GMRT that made these observations possible. GMRT is run by the National Centre for Radio Astrophysics of the Tata Institute of 


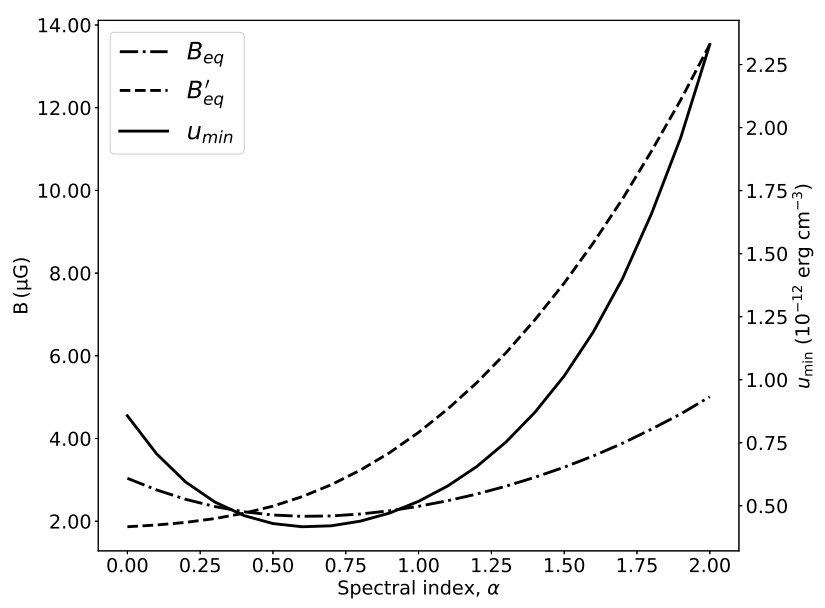

Figure 7. The minimum energy and magnetic fields versus the integrated spectral index are shown. For the relic A4038 the integrated spectral index is 1.5 at the low frequency end. The magnetic field under the assumption of equipartition using fixed frequency interval $\left(B_{\text {eq }}\right)$ and fixed minimum Lorentz factor $\left(B_{\text {eq }}^{\prime}\right)$ are 3.3 and $7.8 \mu \mathrm{G}$, respectively.

Fundamental Research. This research has made use of the NASA/IPAC Extragalactic Database (NED) which is operated by the Jet Propulsion Laboratory, California Institute of Technology, under contract with the National Aeronautics and Space Administration. This research made use of Astropy, a community-developed core Python package for Astronomy (Astropy Collaboration, 2018). The scientific results reported in this article are based in part on data obtained from the Chandra Data Archive.

\section{REFERENCES}

Beck R., Krause M., 2005, Astronomische Nachrichten, 326, 414 Brienza M., et al., 2017, A\&A, 606, A98

Brüggen M., Bykov A., Ryu D., Röttgering H., 2012, Space Sci. Rev., 166, 187

Brunetti G., 2011, MMSAI, 82, 515

Brunetti G., Jones T. W., 2014, International Journal of Modern Physics D, 23, 30007

Brunetti G., Lazarian A., 2016, MNRAS, 458, 2584

Brunetti G., Setti G., Comastri A., 1997, A\&A, 325, 898

Brunetti G., Blasi P., Cassano R., Gabici S., 2004, MNRAS, 350,1174

Cohen A. S., Clarke T. E., 2011, AJ, 141, 149

Duffy P., Blundell K. M., 2012, MNRAS, 421, 108

Dwarakanath K. S., Kale R., 2009, ApJ, 698, L163

Enßlin T. A., Brüggen M., 2002, MNRAS, 331, 1011

Enßlin T. A., Gopal-Krishna 2001, A\&A, 366, 26

Enßlin T. A., Biermann P. L., Klein U., Kohle S., 1998, A\&A, 332,395

Feretti L., Bacchi M., Slee O. B., Giovannini G., Govoni F., Andernach H., Tsarevsky G., 2006, MNRAS, 368, 544

Feretti L., Giovannini G., Govoni F., Murgia M., 2012, AApR, 20,54

Govoni F., Feretti L., 2004, International Journal of Modern Physics D, 13,1549

Gupta Y., et al., 2017, Current Science, 113, 707

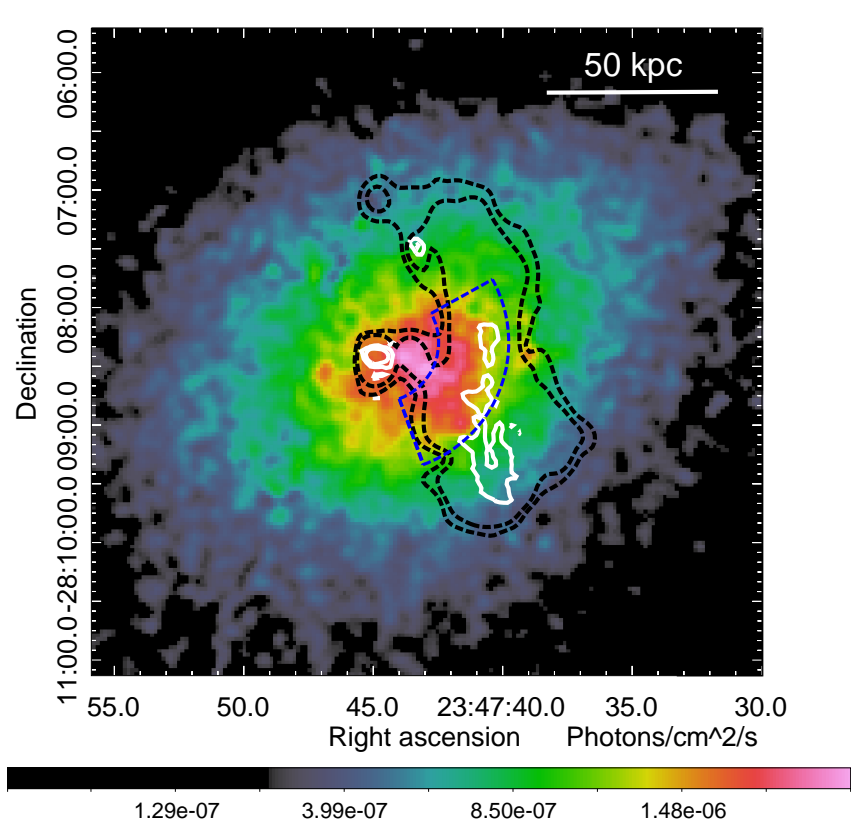

Figure 8. The X-ray image smoothed to $5^{\prime \prime}$ resolution is shown in color scale. The dashed black contours are at $402 \mathrm{MHz}$ (1.0 and $\left.2.0 \mathrm{mJy}^{\mathrm{beam}}{ }^{-1}\right)$. The white contours are at $1.26 \mathrm{GHz}(0.18$ and $1.0 \mathrm{mJy}$ beam $^{-1}$ ). The blue dashed region marks the arc-shaped extension of the central bright elongated region in the X-ray image. The Arc region of the relic appears to skirt the X-ray arc and the Bridge is cospatial with the central elongated region in X-rays.

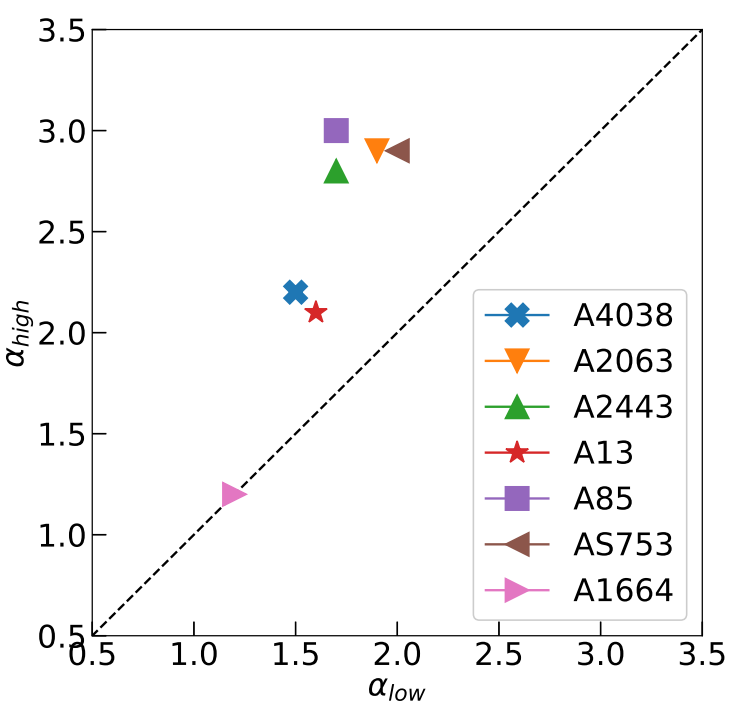

Figure 9. The high versus low frequency spectral indices for the remnant relics sample described in Table 4 are shown. 
Table 3. Spectral properties of the regions (Sec 5).

\begin{tabular}{cccccc}
\hline $\begin{array}{c}\text { Region } \\
\text { number }\end{array}$ & $\alpha_{\text {low }}$ & $\mathrm{B}$ & $\alpha_{\text {high }}$ & $\mathrm{C}$ & $\begin{array}{c}\Delta \alpha \\
\left(\alpha_{\text {high }}-\alpha_{\text {low }}\right)\end{array}$ \\
\hline 0 & $2.6 \pm 0.5$ & $20.2 \pm 4.0$ & - & - & - \\
1 & $3.7 \pm 0.5$ & $29.2 \pm 4.7$ & - & - & - \\
2 & $2.6 \pm 0.6$ & $19.7 \pm 5.3$ & - & - & - \\
3 & $2.1 \pm 0.6$ & $16.0 \pm 4.8$ & - & - & - \\
4 & $2.2 \pm 0.8$ & $16.7 \pm 6.9$ & $3.1 \pm 0.1$ & $24.4 \pm 1.1$ & $0.9 \pm 0.8$ \\
5 & $1.9 \pm 0.1$ & $14.4 \pm 1.2$ & $2.8 \pm 0.2$ & $22.7 \pm 1.5$ & $0.9 \pm 0.2$ \\
6 & $1.9 \pm 0.1$ & $15.0 \pm 1.0$ & $2.8 \pm 0.2$ & $23.0 \pm 1.5$ & $0.9 \pm 0.2$ \\
7 & $1.4 \pm 0.1$ & $10.5 \pm 1.0$ & $2.7 \pm 0.2$ & $22.3 \pm 1.6$ & $1.4 \pm 0.2$ \\
8 & $1.2 \pm 0.2$ & $9.2 \pm 2.1$ & $2.8 \pm 0.2$ & $23.0 \pm 2.2$ & $1.6 \pm 0.3$ \\
9 & $1.6 \pm 0.2$ & $12.1 \pm 1.7$ & $3.0 \pm 0.2$ & $24.5 \pm 2.0$ & $1.3 \pm 0.3$ \\
10 & $1.7 \pm 0.2$ & $13.3 \pm 1.6$ & $2.5 \pm 0.2$ & $19.5 \pm 2.1$ & $0.8 \pm 0.3$ \\
11 & $1.8 \pm 0.2$ & $14.1 \pm 2.0$ & $2.6 \pm 0.2$ & $21.7 \pm 2.1$ & $0.8 \pm 0.3$ \\
12 & $2.3 \pm 0.2$ & $18.5 \pm 1.7$ & $2.3 \pm 0.2$ & $18.4 \pm 2.2$ & $-0.0 \pm 0.3$ \\
13 & $2.1 \pm 0.2$ & $16.5 \pm 1.7$ & $2.6 \pm 0.2$ & $20.5 \pm 2.1$ & $0.5 \pm 0.3$ \\
14 & $2.2 \pm 0.5$ & $16.5 \pm 3.9$ & $2.6 \pm 0.3$ & $20.4 \pm 2.3$ & $0.4 \pm 0.5$ \\
\hline
\end{tabular}

Table 4. Summary of remnant relics from the literature. Col. 1: Cluster name; Col. 2: Redshift; Col. 3: Distance from the cluster center; Col. 4: Low frequency spectral index with the frequency range given in GHz; Col. 5: High frequency spectral index with the frequency range given in GHz; Col. 6: Curvature defined as the difference between the high and low frequency spectral indices. Note: The typical error on the reported spectral indices is $0.1-0.2$.

\begin{tabular}{ccccccc}
\hline Name & $\mathrm{z}$ & $\begin{array}{c}\text { Rcc } \\
(\mathrm{Mpc})\end{array}$ & $\begin{array}{c}\alpha_{\text {low }} \\
(\mathrm{GHz}, \mathrm{GHz})\end{array}$ & $\begin{array}{c}\alpha_{\text {high }} \\
(\mathrm{GHz}, \mathrm{GHz})\end{array}$ & $\begin{array}{c}\Delta \alpha \\
\text { Curvature }\end{array}$ & Reference \\
\hline A4038 & 0.02819 & 0.02 & $1.5(0.074,0.327)$ & $2.2(0.327,1.4)$ & 0.7 & Kale \& Dwarakanath $(2012)$ \\
A2063 & 0.0349 & 0.04 & $1.9(0.08,0.408)$ & $2.9(0.408,1.465)$ & 1.0 & Komissarov \& Gubanov $(1994)$ \\
A2443 & 0.1080 & 0.23 & $1.7(0.074,0.325)$ & $2.8(0.325,1.4)$ & 1.1 & Cohen \& Clarke (2011) \\
A13 & 0.0943 & 0.2 & $1.6(0.08,0.327)$ & $2.1(0.327,1.4)$ & 0.5 & Slee et al. (2001) \\
A85 & 0.0551 & 0.43 & $1.7(0.08,0.327)$ & $3.0(0.327,1.4)$ & 1.3 & Slee et al. (2001) \\
A548b-NW & 0.0424 & 0.43 & - & $\sim 2(1.36,14.3)$ & - & Feretti et al. $(2006)$ \\
A548b-N & 0.0424 & 0.5 & - & $2(1.36,14.3)$ & - & Feretti et al. $(2006)$ \\
AS753 & 0.014 & 0.41 & $2.0(0.33,1.398)$ & $2.9(1.398,2.378)$ & 0.9 & Subrahmanyan et al. (2003) \\
A1664 & 0.1283 & 1.03 & $1.2(0.15,1.4)$ & $1.2(0.15,1.4)$ & 0.0 & Kale \& Dwarakanath $(2012)$ \\
A2048 & 0.0972 & 0.33 & $-(0.074,0.610)$ & $-(0.61,1.4)$ & 1.6 & van Weeren et al. (2011a) \\
\hline
\end{tabular}
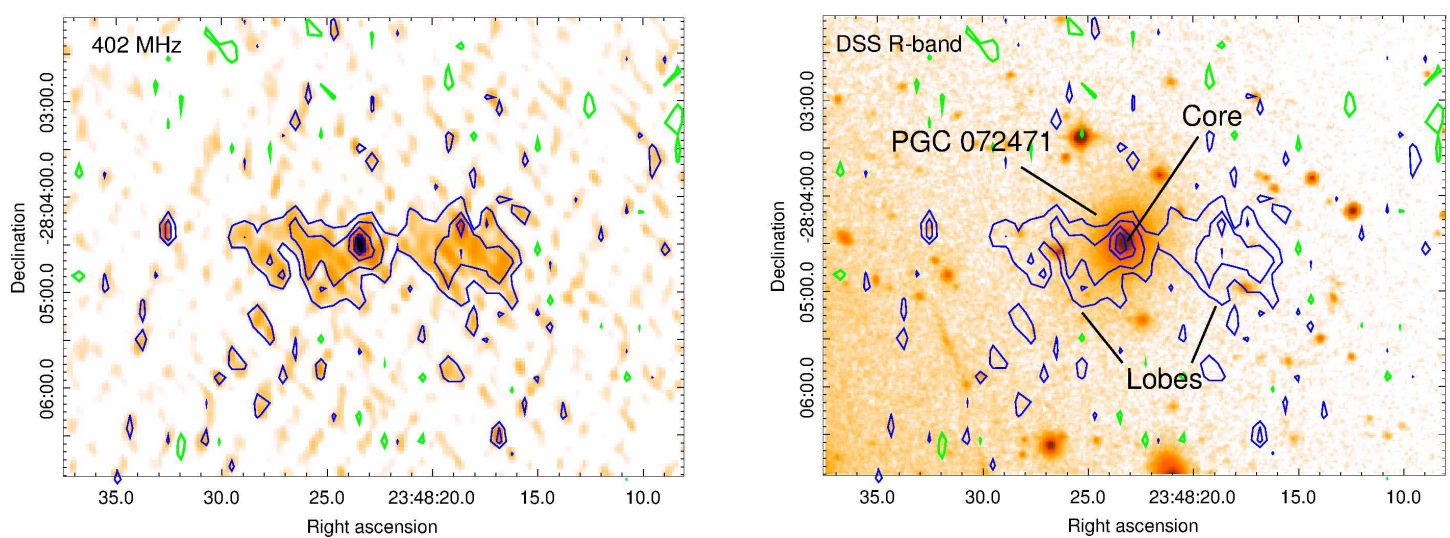

Figure 10. Left:- The $402 \mathrm{MHz}$ image zoomed-in on the rectangle (unrelated to the A4038 relic) in Fig.1 is shown in color and contours. The contours are at $-0.1,0.1,0.2,0.4, \ldots \mathrm{mJy}_{\text {beam }}^{-1}$. The positive contours are blue and the negative are green. Right:- The Digitized Sky Survey R-band image is shown in color with the contours from the left panel overlaid. The compact core can be identified with the galaxy PGC 072471 detected in optical. The lobes are detected for the first time. 
Jaffe W. J., 1977, ApJ, 212, 1

Kale R., Dwarakanath K. S., 2012, ApJ, 744, 46

Kang H., Jones T. W., 2007, Astroparticle Physics, 28, 232

Kempner J. C., Blanton E. L., Clarke T. E., Enßlin T. A., Johnston-Hollitt M., Rudnick L., 2004, in Reiprich T., Kempner J., Soker N., eds, The Riddle of Cooling Flows in Galaxies and Clusters of galaxies. (arXiv: astro-ph/0310263)

Komissarov S. S., Gubanov A. G., 1994, A\&A, 285, 27

McMullin J. P., Waters B., Schiebel D., Young W., Golap K., 2007, in Shaw R. A., Hill F., Bell D. J., eds, Astronomical Society of the Pacific Conference Series Vol. 376, Astronomical Data Analysis Software and Systems XVI. p. 127

Mittal R., Hicks A., Reiprich T. H., Jaritz V., 2011, A\&A, 532, A 133

Nuza S. E., Gelszinnis J., Hoeft M., Yepes G., 2017, MNRAS, 470,240

Offringa A. R., van de Gronde J. J., Roerdink J. B. T. M., 2012, A\&A, 539, A95

Perley R. A., Butler B. J., 2013, ApJS, 204, 19

Petrosian V., 2001, ApJ, 557, 560

Pinzke A., Oh S. P., Pfrommer C., 2013, ArXiv eprints:1301.5644,

Pinzke A., Oh S. P., Pfrommer C., 2017, MNRAS, 465, 4800

Planck Collaboration et al., 2016, A\&A, 594, A27

Sanders J. S., Fabian A. C., Smith R. K., 2011, MNRAS, 410,1797

Schlickeiser R., Sievers A., Thiemann H., 1987, A\&A, 182, 21

Shulevski A., et al., 2015, A\&A, 583, A89

Shulevski A., et al., 2017, A\&A, 600, A65

Slee O. B., Siegman B. C., 1983, Proceedings of the Astronomical Society of Australia, 5, 114

Slee O. B., Roy A. L., Murgia M., Andernach H., Ehle M., 2001, AJ, 122, 1172

Stein P., 1996, A\&ApS, 116, 203

Subrahmanyan R., Beasley A. J., Goss W. M., Golap K., Hunstead R. W., 2003, AJ, 125, 1095

Trasatti M., Akamatsu H., Lovisari L., Klein U., Bonafede A., Brüggen M., Dallacasa D., Clarke T., 2015, A\&A, 575, A45

Wilber A., et al., 2018, MNRAS,

van Weeren R. J., Röttgering H. J. A., Brüggen M., 2011a, A\&A, 527, A114

van Weeren R. J., Brüggen M., Röttgering H. J. A., Hoeft M., Nuza S. E., Intema H. T., 2011b, A\&A, 533, A35

van Weeren R. J., et al., 2016, ApJ, 818, 204

van Weeren R. J., et al., 2017, Nature Astronomy, 1, 0005

\section{APPENDIX A: DETECTION OF DIFFUSE LOBES OF A RADIO GALAXY}

We have detected faint diffuse lobes around a compact source in our uGMRT $402 \mathrm{MHz}$ image. The compact source is associated with the galaxy PGC072471 that is located at the redshift of $0.034130 \pm 0.000067$ (Stein 1996). The diffuse lobes extend nearly symmetrically on the east and west of the compact source. The optical and radio images are shown in Fig. 10. The compact core has a flux density of $2.6 \mathrm{mJy}$ and total flux density including the lobes is $26.6 \mathrm{mJy}$. These are not corrected for the effect of the primary beam. The distance of this source from the phase centre is $10^{\prime}$. The east-west extent of the lobes is 193" which is equal to 109 kpc at the redshift of the host galaxy.

This paper has been typeset from a $\mathrm{T}_{\mathrm{E}} \mathrm{X} / \mathrm{LAT}_{\mathrm{E}} \mathrm{X}$ file prepared by the author. 